\title{
A post-stem cell transplant risk score for Philadelphia-negative acute lymphoblastic leukemia
}

\section{Dietger Niederwieser}

\begin{abstract}
University of Leipzig; Lithuanian University of Health Sciences, Kaunas, Lithuania and Aichi Medical University, School of Medicine, Nagakute, Japan

E-mail: DIETGER NIEDERWIESER - dietger@medizin.uni-leipzig.de
\end{abstract}

doi:10.3324/haematol.2019.246322

$\mathrm{R}$ ecent decades have seen major advances in understanding the genetic basis of hematologic and nonhematologic malignancies. The discovery of the Philadelphia chromosome (Ph) in chronic myeloid leukemia was a key step forward. ${ }^{1,2}$ Since then, many recurrent chromosomal abnormalities, such as $\mathrm{t}(8,21)$ and $t(15 ; 17)$, have been found in acute leukemias, paving the way for identification of altered genes. ${ }^{3}$ These ongoing discoveries have provided and continue to provide major insights into the mechanisms by which key transcription factors and epigenetic modulators regulate normal hematopoiesis and, if dysregulated, promote leukemic transformation. To date, more than 200 balanced chromosomal rearrangements (translocations, insertions and inversions) defining biologically distinct subsets of acute leukemia have been identified. Chromosome analysis, together with molecular determinations, are now important components of routine clinical practice and essential for appropriate diagnosis. Cytogenetic findings have in addition been repeatedly shown to be among the most important and independent prognostic factors in both acute myeloid leukemia and acute lymphoblastic leukemia (ALL).,5 For all these reasons, specific chromosome alterations and their molecular counterparts have been included in the World Health Organization classification of hematologic malignancies and together with morphology, immunophenotype and clinical features are used to define distinct disease entities. ${ }^{6}$

The first comprehensive cytogenetic analysis showing biological and prognostic significance in adult ALL was performed at the Third International Workshop on Chromosomes in Leukemia in 1981. The frequency of abnormal karyotypes was shown to be slightly higher in adult than in pediatric ALL $(60-69 \%$ vs. $58-64 \%$, respectively) with $\mathrm{t}(9 ; 22)(\mathrm{q} 34 ; \mathrm{q} 11)$ being the most frequent translocation. ${ }^{8}$ Less than $6 \%$ of children, but up to $40 \%$ of adults $\geq 40$ years of age, with ALL harbor a Ph translocation $\left(\mathrm{Ph}^{+}\right)$with or without additional alterations, which is a poor prognostic feature regardless of age. In contrast, less than $12 \%$ of adults, but $25 \%$ of children have high hyperdiploidy, a good prognostic feature. ${ }^{4}$

One of the hurdles to developing a more sophisticated cytogenetic profile is the overall incidence and, in particular, the different subsets of $\mathrm{Ph}^{-}$adult ALL, each of which accounts for less than $10 \%$ of the total. Only sparse information is available on Ph ALL patients. The most frequent $\mathrm{Ph}$ - chromosomal aberrations include $\mathrm{t}(4 ; 11)(\mathrm{q} 21 ; \mathrm{q} 23) / K M T 2 A-A F F I$ (3-7\%) involving the $M L L$ gene, translocations $\mathrm{t}(8 ; 14)(\mathrm{q} 24 ; \mathrm{q} 11)(2 \%)$ involving $m y c$, $\mathrm{t}(1 ; 19)(\mathrm{q} 23 ; \mathrm{p} 13) / T C F 3-P B X 1 \quad(2-3 \%), \mathrm{t}(10 ; 14)(\mathrm{q} 24 ; \mathrm{q} 11)$ $(2 \%), t(11 ; 19)(q 23 ; 13.3)$ and structural abnormalities such as $9 p, 6 q$, and $12 p, 18,19$. Further cytogenetic changes include the multiaberrant karyotype, monosomy 7 , monosomy $9,+8$, del11 and low hypodiploidy, near triploidy and high hyperdiploidy. ALL study groups, including the Medical Research Council (MRC), Eastern Cooperative Oncology Group (ECOG), Southwest Oncology Group (SWOG), Northern Italy Leukemia Group (NILG), North UK and Gruppo Italiano Malattie Ematologiche dell'Adulto (GIMEMA) categorize the cytogenetic alterations at diagnosis into risk groups. Unfortunately, the representation of patients treated with hematopoietic stem cell transplantation (SCT) is limited in these analyses. The largest study with patients undergoing allogeneic SCT was the MRCECOG study with 310 patients. ${ }^{9}$ Here, four risk categories were identified using the modified MRC-ECOG score (very high, high, intermediate and standard). ${ }^{10}$

In a study reported by Aleksandr Lazaryan et al. in this issue of Haematologica, the Acute Leukemia Working Committee of the Center for International Blood and Marrow Transplant Research (CIBMTR) investigated the usefulness of the MRC-ECOG score in a large cohort of patients after SCT $(n=1731)$ all of whom were adults with $\mathrm{Ph}$ ALL. ${ }^{11}$ While the standard- risk group had favorable outcomes compared to the intermediate-risk group, the adverse risk group was not clearly inferior using the modified MRC-ECOG score. The analysis of relapse and posttransplant treatment failure revealed that $\mathrm{t}(8 ; 14)$, monosomy 7 and complex karyotype were the major important determinants. As a consequence, the authors propose, in addition to the modified MRC-ECOG score at diagnosis, the CIBMTR risk score for transplant, which does not include the $t(4 ; 11), t(1 ; 19), t(17 ; 19), t(5 ; 14)$ and +8 , but does include $t(8 ; 14), t(11 ; 19)$, monosomy 7 , del(7q), del(11q) and complex karyotype (Figure 1).

Previous studies on ALL patients after SCT have concluded that cytogenetics do not predict overall survival. The difference in respect to the current study might be explained by the high number of patients transplanted in advanced phase disease in addition to the high number of patients with $\mathrm{Ph}^{+}$ALL included in one study. ${ }^{12}$ Another study found no difference in overall survival between patients with high risk [defined as $\mathrm{t}(4 ; 11)(\mathrm{q} 21 ; \mathrm{q} 23)$, $\mathrm{t}(8 ; 14)(\mathrm{q} 24 ; 32)$, low hypodiploidy, complex karyotype] and standard-risk cytogenetics, most probably as a consequence of the low number of patients in the high-risk group. ${ }^{13}$ A different study identified $\mathrm{t}(4 ; 11) / K M T 2 A-A F F 1$ and $\mathrm{t}(\mathrm{v} ; 14 \mathrm{q} 32) / I G H$ in $\mathrm{Ph}$ patients, but censored patients at the time of SCT. ${ }^{14}$

A source of uncertainty in the current analysis is the lack of information on cytogenetic results at transplant. ${ }^{15}$ Furthermore, molecular information at diagnosis and minimal residual disease might have influenced the results of this retrospective analysis. Despite these flaws, the results 
Risk Group

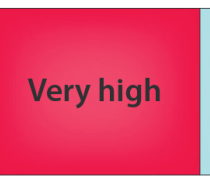

- $t(4 ; 11), t(8 ; 14)$

- complex karytype

- low hypodiploidy (30-39)/

near triploidy (60-78)
Risk Group

Modified MRC-ECOG

(Pullarkat et al. Blood 2008)
CIBMTR risk score in Ph neg ALL (Lazaryan et al. Haematologica 2020)
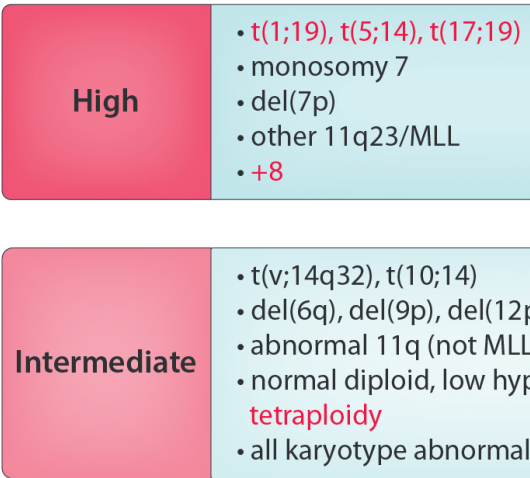

t(v;14q32), t(10;14)

- del(6q), del(9p), del(12p), del(17p)

- abnormal 11q (not MLL)

- normal diploid, low hyperdiploidy (47-50), tetraploidy - all karyotype abnormalities not identified

Standard
• high hyperdiploidy
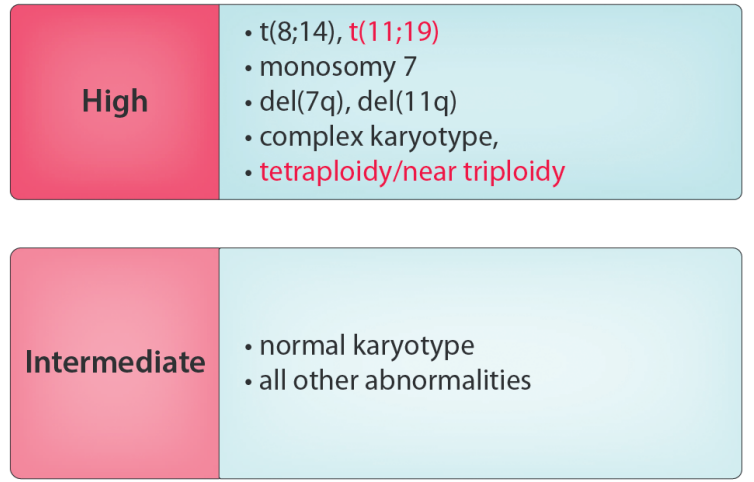

Favorable

Figure 1. Comparison of two cytogenetic risk classifications for Philadelphia chromosome-negative acute lymphoblastic leukemia. The modified Medical Research Council - Eastern Cooperative Oncology Group (MRC-ECOG) score at diagnosis versus the Center for International Blood and Marrow Transplant Research (CIBMTR) risk score for post-transplant Philadelphia chromosome-negative acute lymphoblastic leukemia. Differences between the risk scores are shown in red.

are derived from the largest cohort of $\mathrm{Ph}$ patients treated with SCT to date and show clear distinctions in leukemiafree survival (LFS) using just three risk groups.

Several aspects of the article by Lazaryan et al. ${ }^{11}$ are of interest. Post-transplant risk scores differ from those for patients treated with conventional therapy. This may be a consequence of graft-versus-tumor susceptibility. It is interesting to see that translocations, except for $\mathrm{t}(8 ; 14)$ and $\mathrm{t}(1 ; 19)$, are noticeably absent from the CIBMTR risk score compared to the modified MRC-ECOG risk score (Figure 1). While $\mathrm{t}(1 ; 19)(\mathrm{q} 23 ; \mathrm{p} 13), \quad \mathrm{t}(4 ; 11)(\mathrm{q} 21 ; \mathrm{q} 23)$, $\mathrm{t}(5 ; 14)(\mathrm{q} 35 ; \mathrm{q} 32)$ and $\mathrm{t}(17 ; 19)(\mathrm{q} 22 ; \mathrm{p} 13)$ were identified as risk factors at diagnosis/before SCT, they were not considered to be adverse post-SCT. It is feasible that the abnormal proteins produced by translocations may directly or indirectly affect malignant cell immunogenicity and enhance the graft-versus-tumor effect.

The translocations $t(8 ; 14)$ and $t(11 ; 19)$ still remain in the high-risk category. A possible reason might be the association of $\mathrm{t}(8 ; 14)$ with the involvement of the myc gene on chromosome 8 and of $\mathrm{t}(11 ; 19)$ with the MLL gene, underlying the prevalence of tumor-specific rather than immunogenic factors.

Multiple mechanisms have been proposed to be responsible for the high relapse rate in diseases with monosomy 7 and complex karyotype, including loss of tumor suppressor genes, haplo-insufficiency or loss of IKZF1. These alterations may be less susceptible to graft-versus-tumor reactions. The results are similar to those previously seen in acute myeloid leukemia, in which $\mathrm{t}(11 ; 19),{ }^{16-18}$ monosomy 7 , deletion $7 q^{19,20}$ and complex karyotype are also risk factors and play an important role in outcome. Similar mechanisms might therefore influence relapse rates after SCT in both acute myeloid leukemia and ALL.

A further consequence of the results of the analysis by Lazaryan et al. is the evident need to reduce the relapse rate in high-risk (but also in normal-risk) patients. This may be possible by evaluating minimal residual disease before and after SCT. The important role of minimal residual disease in predicting outcome at an individual level has recently been published. ${ }^{21}$ Optimizing SCT outcome by tailoring immunosuppression in the early phase, in response to post-transplant monitoring of disease-specific minimal residual disease or chimerism would be an appropriate approach. The relapse risk in $\mathrm{Ph}$ ALL may be reduced by new drugs, such as blinatumomab, inotuzum$\mathrm{ab}$ ozogamycin or tisagenlecleucel. In this context, the results presented by Lazaryan et al. should provide a stimulus for prospective clinical studies.

Furthermore, those translocations associated with implied susceptibility to graft-versus-tumor reactions may provide a lead for the identification of immunogenic tumor-specific antigens, while all translocations are potential targets for small molecules able to neutralize diseasespecific products, such as driver kinases or activation pathways. In patients with deletions or monosomy such efforts might be difficult.

Finally, scores might be influenced by the different treat- 
ment possibilities available today. Often large datasets are collected over a long time with considerable changes in first-line therapy, such as introduction of pediatric-based regimens, while the number of patients in different categories remains small. Considering the relative homogeneity of transplant procedures in comparison to the different non-transplant protocols, the post-transplant CIBMTR score represents an important prognostic tool.

\section{References}

1. Nowell P, Hungerford D. A minute chromosome in human chronic granulocytic leukemia. Science. 1960;132:1497.

2. Rowley JD. A new consistent chromosomal abnormality in chronic myelogenous leukaemia identified by quinacrine fluorescence and Giemsa staining. Nature. 1973;243(5405):290-293.

3 . Rowley JD. Identificaton of a translocation with quinacrine fluorescence in a patient with acute leukemia. Ann Genet. 1973. 1973;16(2):109-112.

4. Mrózek K, Heerema NA, Bloomfield CD. Cytogenetics in acute leukemia. Blood Rev. 2004;18(2):115-136.

5. Rowe JM, Buck G, Burnett AK, et al. Induction therapy for adults with acute lymphoblastic leukemia: results of more than 1500 patients from the international ALL trial: MRC UKALL XII/ECOG E2993. Blood. 2005;106(12):3760-3767.

6. Döhner H, Estey E, Grimwade D, et al. Diagnosis and management of AML in adults: 2017 ELN recommendations from an international expert panel. Blood. 2017;129(4):424-447.

7. Mittelman F. The Third International Workshop on Chromosomes in Leukemia. Lund, Sweden, July 21-25, 1980. Introduction. Cancer Genet Cytogenet. 1981(4):96-98.

8. Roberts KG. Genetics and prognosis of ALL in children vs adults. Hematology Am Soc Hematol Educ Program. 2018;2018(1):137-145.

9. Moorman AV, Harrison CJ, Buck GAN, et al. Karyotype is an independent prognostic factor in adult acute lymphoblastic leukemia (ALL): analysis of cytogenetic data from patients treated on the Medical Research Council (MRC) UKALLXII/Eastern Cooperative Oncology Group (ECOG) 2993 trial. Blood. 2007;109(8):3189-3197.

10. Pullarkat V, Slovak ML, Kopecky KJ, Forman SJ, Appelbaum FR. Impact of cytogenetics on the outcome of adult acute lymphoblastic leukemia: results of Southwest Oncology Group 9400 study [eng] Blood. 2008;111(5):2563-2572.

11. Lazaryan A, Dolan M, Zhang M-J, et al. Impact of cytogenetic abnor malities on outcomes of adult Philadelphia-negative acute lymphoblastic leukemia after allogeneic hematopoietic stem cell transplantation: a study by the Acute Leukemia Working Committee of the Center for International Blood and Marrow Transplant Research [eng]. Haematologica. 2020;105(5):1329-1338.

12. Aldoss I, Tsai N-C, Slovak ML, et al. Cytogenetics does not impact outcomes in adult patients with acute lymphoblastic leukemia undergoing allogeneic hematopoietic cell transplantation. Biol Blood Marrow Transplant. 2016;22(7):1212-1217.

13. Shimizu H, Doki N, Kanamori H, et al. Prognostic impact of cytogenetic abnormalities in adult patients with Philadelphia chromosomenegative ALL who underwent an allogeneic transplant. Bone Marrow Transplant. 2019;54(12):2020-2026.

14. Lafage-Pochitaloff $M$, Baranger L, Hunault $M$, et al. Impact of cytogenetic abnormalities in adults with $\mathrm{Ph}$-negative B-cell precursor acute lymphoblastic leukemia. Blood. 2017;130(16):1832-1844.

15. Niederwieser C, Nicolet D, Carroll AJ, et al. Chromosome abnormalities at onset of complete remission are associated with worse outcome in patients with acute myeloid leukemia and an abnormal karyotype at diagnosis: CALGB 8461 (Alliance). Haematologica. 2016;101(12):1516-1523

16. Döhner H, Weisdorf DJ, Bloomfield CD. Acute myeloid leukemia. N Engl J Med. 2015;373(12):1136-1152

17. Grimwade D, Hills RK, Moorman AV, et al. Refinement of cytogenetic classification in acute myeloid leukemia: determination of prognostic significance of rare recurring chromosomal abnormalities among 5876 younger adult patients treated in the United Kingdom Medical Research Council trials. Blood. 2010;116(3):354-365.

18. Chen Y, Kantarjian H, Pierce S, et al. Prognostic significance of $11 \mathrm{q} 23$ aberrations in adult acute myeloid leukemia and the role of allogeneic stem cell transplantation. Leukemia. 2013;27(4):836-842.

19. Poiré X, Labopin M, Polge E, et al. The impact of concomitant cytogenetic abnormalities on acute myeloid leukemia with monosomy 7 or deletion $7 \mathrm{q}$ after HLA-matched allogeneic stem cell transplantation. Am J Hematol. 2020;95(3):282-294.

20. Inaba $\mathrm{T}$, Honda $\mathrm{H}$, Matsui $\mathrm{H}$. The enigma of monosomy 7 . Blood. 2018;131(26):2891-2898.

21. Shah MV, Jorgensen JL, Saliba RM, et al. Early post-transplant minimal residual disease assessment improves risk stratification in acute myeloid leukemia 7. Biol. Blood Marrow Transplant. 2018;24(7): 1514-1520. 